\title{
Empirical relations among scattering, roughness parameters, and thickness of aluminum films
}

\author{
J. I. Larruquert, J. A. Méndez, and J. A. Aznárez
}

\begin{abstract}
Experimental measurements of the angular distribution of scattering and scanning electron microscopy pictures of thin aluminum films were used to relate the total integrated scattering and the statistical parameters of the surface roughness to the film thickness.

Key words: Scattering, aluminum, roughness.
\end{abstract}

\section{Introduction}

Aluminum mirrors reflect electromagnetic radiation from the microwave to the far-ultraviolet (FUV) regions. Their surface shows a short-scale roughness that results in a certain decay of the specular reflectance and the appearance of scattered light. This degradation increases rapidly from the visible to the UV region.

At the moment we are initiating the preparation of ultrahigh-vacuum-deposited aluminum mirrors in order to measure their FUV reflectance with special attention being paid to the surface roughness that is required. To obtain a first approach, we started with a preliminary study on scattering in the visible spectral range of high vacuum-deposited aluminum films. In the future we expect to complete this study in the FUV.

The roughness of a thin aluminum film is determined by many factors ${ }^{1-3}$ such as the deposition method, the rate and angle of deposition, the thickness of the layer, the type of substrate, its roughness, its temperature during deposition, the surrounding atmosphere during deposition, and aging. In this paper we study the dependence of the total integrated scattering (TIS) and the surface-roughness parameters of aluminum mirrors versus film thickness, keeping as a constant the rest of the parameters mentioned above.

By observing scanning electron microscopy (SEM) pictures of aluminum films, we get information on the lateral size of aluminum crystals. Measure-

The authors are with Consejo Superior Investigaciones Cientificas, Instituto de Optica, C/Serrano 121, 28006 Madrid, Spain.

Received 5 March 1992.

0003-6935/93/316341-06\$06.00/0.

(C) 1993 Optical Society of America. ments of the angular distribution of scattering of each mirror with visible light are used to calculate the surface-roughness parameters and the TIS.

\section{Relationship between the Angular Distribution of Scattering and Surface-Roughness Parameters}

Mirror surfaces have microirregularities that give rise to scattered light from the specular direction. The surface profile determines the angular distribution of scattering and TIS.

In order to simplify the description of the profile, we suppose that the height of microirregularities with respect to as the mean plane follows a Gaussian statistical distribution. The roughness of the surface is described by its autocovariance function $G$, defined as follows:

$$
G(\boldsymbol{\tau})=\frac{1}{A} \iint_{A} \mathrm{~d} x \mathrm{~d} y h(\mathbf{r}) h(\mathbf{r}+\tau),
$$

where $h$ is the height distribution function of the surface roughness with respect to the mean level and $A$ is the total area whose autocovariance is searched ( $A$ is supposed to be much greater than $|\tau|^{2}$ ).

For thin aluminum films grown on smooth substrates, the scattered intensity is a small fraction of the incident light. Hence, the angular distribution of scattering can be calculated by means of a firstorder perturbation vector scattering theory in terms of the autocovariance function of the surface.

The presence of surface roughness also permits the incident radiation to excite surface polaritons. Partial reradiation of this energy, caused by surface polariton decay, is added to the roughness-induced scattering and masks it., ${ }^{4,5}$ For the films with which we are dealing, the reradiation can be neglected in the calculation of the angular distribution of scattering in 
the visible spectrum for smooth aluminum surfaces since the aluminum surface polariton wavelength is much shorter than the visible wavelength $\left(\lambda_{\mathrm{sp}}=118\right.$ $\mathrm{nm}$ ) and reradiation is a second-order function of the surface-roughness parameters. ${ }^{5}$

Here we reproduce the expression to obtain the normalized first-order perturbation angular distribution of scattering ${ }^{6}$ :

$$
\begin{aligned}
\frac{1}{P_{0}} \frac{\mathrm{d} P}{\mathrm{~d} \Omega}= & \frac{(\omega / c)^{4}}{\pi^{2}} \cos \theta_{0} \cos ^{2} \theta|1-\epsilon|^{2} \\
& \times\left(\frac{\left|\chi_{\theta}\right|^{2}}{|\nu-i q \epsilon|^{2}}+\frac{\left|\chi_{\phi}\right|^{2}}{|\nu-i q|^{2}}\right) g\left(\mathbf{k}-\mathbf{k}_{0}\right),
\end{aligned}
$$

where

$$
\begin{aligned}
& \chi_{\theta}=\frac{\left(\nu \nu_{0} \cos \phi+k k_{0} \epsilon\right) \cos \phi^{\prime}}{\nu_{0}-i q_{0} \epsilon}-\frac{i\left(\frac{\omega}{c}\right) \nu \sin \phi \sin \phi^{\prime}}{\nu_{0}-i q_{0}}, \\
& \chi_{\phi}=\left(\frac{\omega}{c}\right)\left[\frac{\nu_{0} \sin \phi \cos \phi^{\prime}}{\nu_{0}-i q_{0} \epsilon}+i \frac{\left(\frac{\omega}{c}\right) \cos \phi \sin \phi^{\prime}}{\nu_{0}-i q_{0}}\right],
\end{aligned}
$$

$P_{0}$ is the incident intensity, $\epsilon$ is the complex dielectric constant, $\theta_{0}$ is the polar angle of incidence with respect to the mean surface normal, $\theta$ and $\phi$ are the polar and azimuthal angles of scattering with respect to the mean surface normal and the plane of incidence, and $\phi^{\prime}$ is the polarization angle of the incident beam relative to the plane of incidence. $k_{0}=(\omega / c) \sin$ $\theta_{0}, q_{0}=(\omega / c) \cos \theta_{0}, k=(\omega / c) \sin \theta$, and $q=(\omega / c) \cos \theta$ are the components of the wave vector parallel and perpendicular to the surface for incident and scattered light. $\nu_{0}=\left[k_{0}^{2}-\epsilon(\omega / c)^{2}\right]^{1 / 2}$ and $\nu=\left[k^{2}-\right.$ $\left.\epsilon(\omega / c)^{2}\right]^{1 / 2}$. $g$ is the power spectral density, that is, the Fourier transform of the autocovariance function.

TIS is obtained by integration of the normalized angular distribution of scattering in the scattering hemisphere:

$$
\mathrm{TIS}=\frac{1}{P_{0}} \int_{\mathrm{SH}}\left(\frac{\mathrm{d} P}{\mathrm{~d} \Omega}\right) \mathrm{d} \Omega .
$$

For symmetry considerations during aluminum film deposition, we assumed the autocovariance function to be isotropic, so that $G(\tau)=G(\tau=|\tau|)$ and $g(\mathbf{k})=$ $g(k=|\mathbf{k}|)$. In addition a Gaussian autocovariance function is assumed ${ }^{7}$ :

$$
G(\tau)=\sigma^{2} \exp \left(-\tau^{2} / T^{2}\right)
$$

where $\sigma$ is the root-mean-square roughness and $T$ is the autocorrelation length. The roughness of a slightly rough surface is described by two parameters: $\sigma$ (gives information of the vertical size of microirregularities) and $T$ (gives their lateral size). The power spectral density is

$$
g(k)=\pi \sigma^{2} T^{2} \exp \left(-k^{2} T^{2} / 4\right) .
$$

\section{Experimental}

\section{A. Preparation of Mirrors}

Aluminum films were deposited in a high vacuum system at a pressure of $\sim 10^{-6}$ Torr. With a tungsten filament, we evaporated aluminum of $99.99 \%$ purity and deposited it onto floated glass substrates placed normal to the direction of the incoming aluminum. A thickness controller monitored the deposition rate and the accumulated thickness. The thicknesses of aluminum films were measured $a$ posteriori by Tolanski interferometry. A thermocouple was used to measure the temperature of the substrate during evaporation. After deposition, the aluminized samples were extracted from the vacuum chamber.

A total of 27 aluminum films were deposited on separate substrates, with a thickness range of 41 to $5000 \mathrm{~nm}$ being covered. The deposition rate was rounded off to $10 \mathrm{~nm} / \mathrm{s}$. The heat radiated by the filament during evaporation slightly increased the final substrate temperature. This increment, $\sim 15^{\circ} \mathrm{C}$ for the thickest films, was considered negligible in the process of modeling the surface profile.

\section{B. Scanning Electron Microscopy Analysis}

In order to describe the roughness of the mirrors, we observed the surface of some of them, chosen with stepped thicknesses, in SEM. Figures 1-3 correspond to 41-, 500-, and 4120-nm film thicknesses, respectively.

We can deduce information about the lateral size of aluminum grain $d$ from them by averaging a large number of grain widths measured on the pictures. The autocorrelation length is related to $d$ according to ${ }^{8}$

$$
T=d / \sqrt{2 \pi} .
$$

C. Measurements of the Angular Distribution of Scattering Figure 4 shows the setup in which the angular distribution of scattering was measured. A 514-nm argon laser light beam was reflected by the aluminum

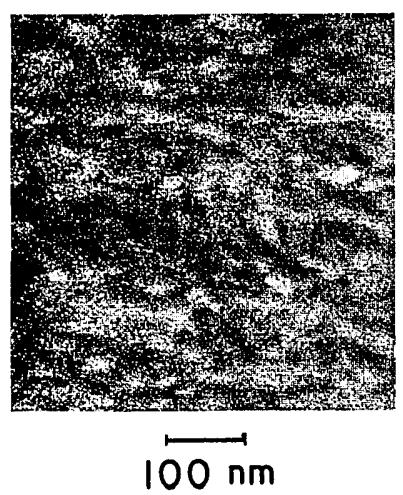

Fig. 1. SEM picture of a 41-nm-thick aluminum film. 


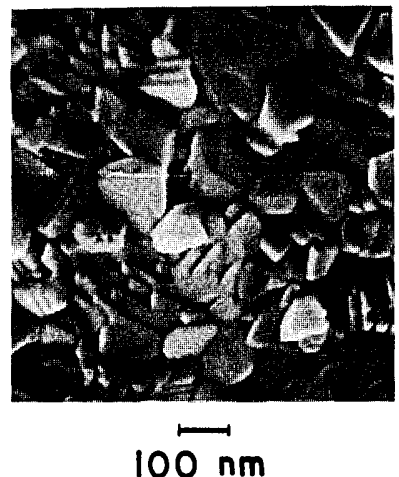

Fig. 2. SEM picture of a 500-nm-thick aluminum film.

mirror at an angle of incidence of $\theta_{0}=5^{\circ}$. The sample mirror $\mathrm{S}$ was mounted on a rotatable sample holder RSH. Detector $\mathrm{D}_{2}$, mounted on a goniometer, was used to measure the light scattered by the mirror for a set of scattering angles in the plane of incidence $\left(\phi=0^{\circ}\right)$. Chopper C and a lock-in amplifier LA were used to increase the signal-to-noise ratio. Another detector $D_{1}$ was used to monitor the intensity variations of the incident beam. Pinhole $P_{1}$ was used to filter the laser beam. The incident beam was polarized perpendicular to the plane of incidence $\left(\phi^{\prime}=90^{\circ}\right)$. The angular distribution of scattering of aluminized samples was measured within four months after deposition.

The angular distribution of scattering was calculated as the scattered intensity measured in each direction divided by the incident beam intensity and by the solid angle intersected by the detector. The scattering angle covered was from $10^{\circ}$ to $50^{\circ}$ away from the specular direction.

\section{Results and Discussion}

\section{A. Calculation of $T$ from Scanning Electron} Microscopy Pictures

Figure 5 shows the values of $T$, calculated from $d$ by Eq. (8), versus thickness. A simple and reasonably good fit can be obtained with the one-parameter function:

$$
T=2.8 \sqrt{e} \quad(e \text { and } T \text { in nanometers }),
$$

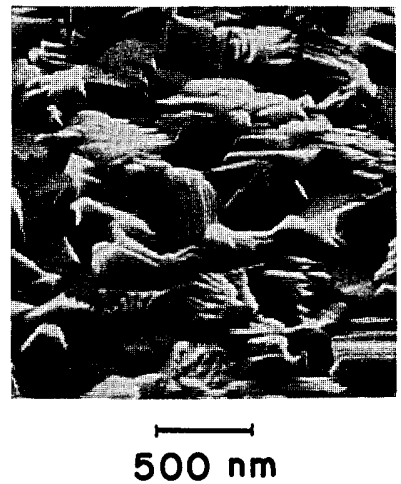

Fig. 3. SEM picture of a $4120-\mathrm{nm}$-thick aluminum film.

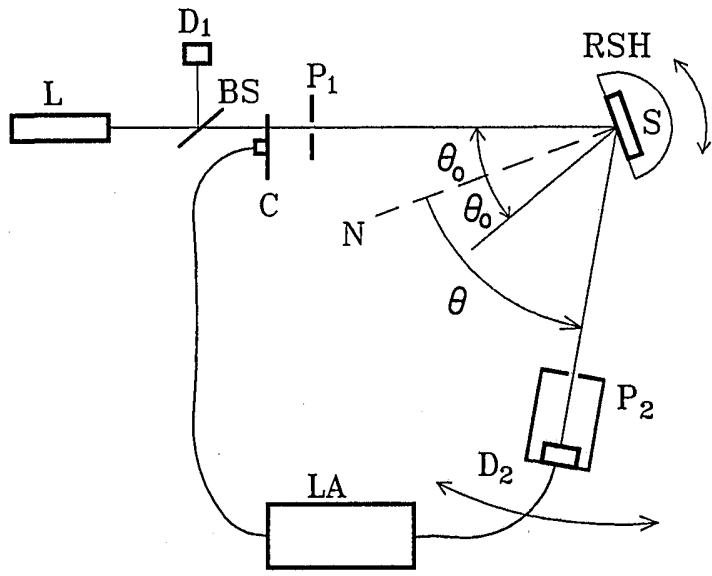

Fig. 4. Setup to measure the angular distribution of scattering: $\mathrm{L}, 515-\mathrm{nm}$ argon laser; $\mathrm{D}_{1}, \mathrm{D}_{2}$, reference and scattering detectors; BS, beam splitter; C, chopper; LA, lock-in amplifier; S, sample; $\mathrm{RSH}$, rotatable sample holder; $\mathrm{P}_{1}, \mathrm{P}_{2}$, apertures; $\mathrm{N}$, mean surface normal; $\theta_{0}$, angles of incidence and of specular reflection; $\theta$, scattering angle.

where $e$ is the aluminum film thickness. The lateral size of aluminum grains grows monotonically with thickness in the whole range. $T$ grows quickly when the thickness of thin films is increased, but the rate of increase is reduced as films become thicker.

B. Calculation of Surface-Roughness Parameters and TIS from Measurements of the Angular Distribution of Scattering

\section{Aluminum/Substrate Effective Parameters}

Equation (2) was used to calculate the surfaceroughness parameters from the angular distribution of scattering of a film. The problem was overdimensioned: a large collection of scattering angular measurements and two unknowns. A Monte Carlo method was employed to search the pair $\{\sigma, T\}$ that

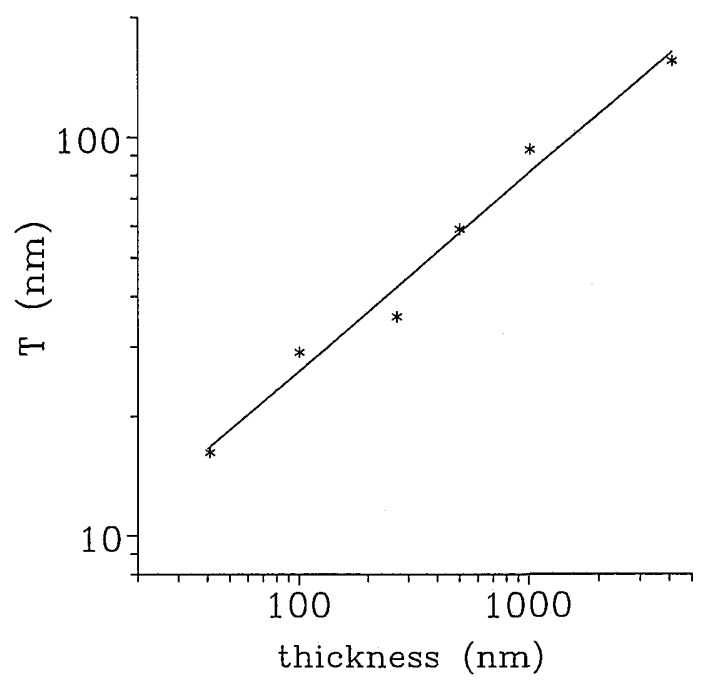

Fig. 5. *, Log-log plot of aluminum autocorrelation length values obtained from SEM pictures versus thickness; solid line, fit of $T$ to the square root of thickness. 
minimized the error function:

$$
r_{j}=\sum_{i}\left[S_{i}-S\left(\theta_{i}, \sigma_{j}, T_{j}\right)\right]^{2},
$$

where $\left\{S_{i}\right\}$ is the set of angular scattering measurements in the directions $\left\{\theta_{i}\right\}, S\left(\theta_{i}, \sigma_{j}, T_{j}\right)$ is the angular scattering calculated in the direction $\theta_{i}$ for the pair $\left\{\sigma_{j}, T_{j}\right\}$ that is being tested, and $r_{j}$ is the error in iteration $j$. Along the iterations, the set $\left\{\sigma_{j}, T_{j}\right\}$ corresponding to the smallest $r_{j}$ was conserved.

Figures 6-8 show $\sigma, T$, and TIS [TIS was calculated from $\sigma$ and $T$ by numerical integration of Eq. (5) and expressed in percentage relative to the incident intensity] versus the thickness of aluminum films. For the thickest films, TIS is too large to be described by a first-order theory, so that the parameters presented for the thickest films should be interpreted as an estimation of the real values.

Values of $\sigma, T$, and TIS obtained were affected by an important dispersion around central values $\sigma_{g}, T_{g}$, and TIS $_{g}$ for thicknesses below $500 \mathrm{~nm}$. This is attributed to the difficulty of controlling all the parameters concerned in the determination of the surface roughness and in the presence of macroirregularities in the surface because of defects, scratches, etc.

However, some interesting deductions can be inferred. Figure 6 shows that the rms roughness tends to a nonzero value at the lowest film thicknesses. In Fig. 7 the autocorrelation length takes a large value at the lowest thicknesses; it diminishes to a minimum when thickness is increased and begins to rise for thicker films. However, in Fig. 5 the autocorrelation length monotonically increases with thickness in the whole thickness range. The explanation of this apparent contradiction is that the substrates were not perfectly smooth. For the thinnest aluminum films for which aluminum crystals were small, the main contribution to scattering was that of the

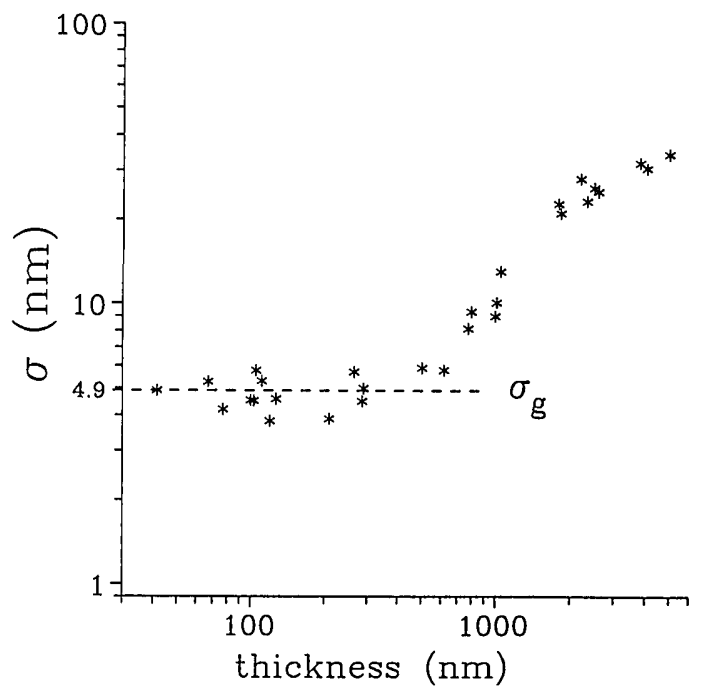

Fig. 6. Log-log plot of the rms roughness of aluminum films on glass substrates versus aluminum thickness. Dashed line, substrate rms roughness.

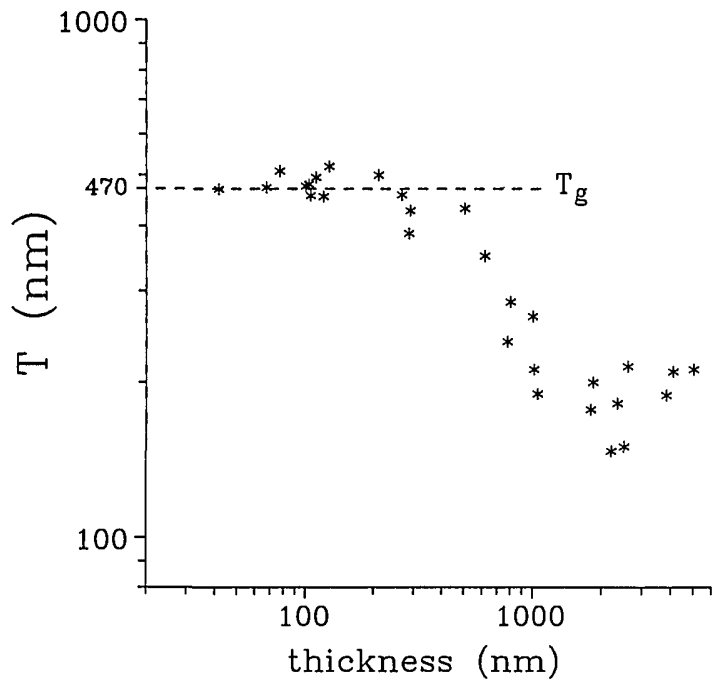

Fig. 7. Plot of the autocorrelation length of aluminum films on glass substrates versus aluminum thickness. Dashed line, substrate autocorrelation length.

substrate roughness. Then the values of $\sigma$ and $T$ at the lowest film thicknesses corresponded to the roughness of the glass substrate. However, since the substrate profile had larger spatial wavelengths and its slope was shorter than those of the aluminum microstructure, the substrates appeared to be smooth on the SEM pictures and the roughness observed corresponded to that of the aluminum film.

Figure 7 can be understood as follows: for thin aluminum films, $T$ of the substrate dominates over that of the aluminum film. As the film thickness increases, the surface becomes rougher and it is no longer negligible in the determination of scattering. Since the autocorrelation length of aluminum crystals is smaller than that of the substrate, the combined effect of both microstructures shows an effective autocorrelation length smaller than that of the

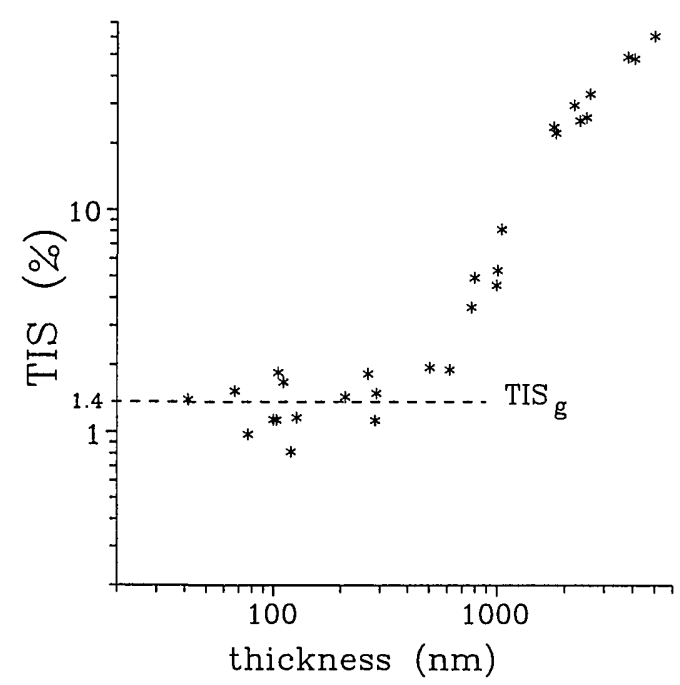

Fig. 8. Log-log plot of TIS of aluminum films on glass substrates versus aluminum thickness. Dashed line, TIS that is due to substrate roughness. 
substrate alone, whereas both $\sigma$ and TIS are larger than those of the substrate. As aluminum films become thicker, the aluminum crystals grow in both dimensions, so that at a certain thickness the scattering corresponding to the aluminum microstructure is greater than that of the substrate. Therefore, after a minimum in the effective autocorrelation length, larger thickness implies larger aluminum crystals and larger autocorrelation length.

The values of $\sigma_{g}$ and $T_{g}$ for the glass substrate can be calculated from the averaged scattering angular distribution of a set with the thinnest films. The calculated values are $\sigma_{g}=4.9 \mathrm{~nm}, T_{g}=470 \mathrm{~nm}$, and the corresponding TIS is $\mathrm{TIS}_{g}=1.4 \%$.

\section{Specific Parameters of Aluminum}

Since the mean slope of the substrate surface, estimated by the ratio $\sigma_{g} / T_{g} \sim 1 \%$, is small and the aluminum crystals are smaller than $T_{g}$, it can be considered that the incoming aluminum sees a locally smooth substrate surface. In this assumption, the roughness of the deposited film can be supposed independent of the substrate roughness. This implies that both height distributions are independent from one another:

$$
h(\mathbf{r})=h_{1}(\mathbf{r})+h_{2}(\mathbf{r}),
$$

where $h_{1}$ is the height distribution of the substrate roughness, independent of $h_{2}$, the height distribution of the aluminum film roughness, and $h$ is the combined height distribution. $h_{1}$ can be raised to the same level as that of the outer aluminum surfaces:

$$
h_{1}{ }^{\prime}(\mathbf{r})=h_{1}(\mathbf{r})+e,
$$

where $e$ is the mean aluminum thickness. We redefine the total height distribution as

$$
h^{\prime}(\mathbf{r})=h_{1}{ }^{\prime}(\mathbf{r})+h_{2}(\mathbf{r}),
$$

where $h^{\prime}$ is a zero mean height distribution obtained by the addition of two zero mean, independent height distributions. This change allows both mean planes to coincide. is

The autocovariance function of the total roughness

$$
G(\tau)=G_{1}(\tau)+G_{2}(\tau),
$$

where $G_{1}$ and $G_{2}$ are the autocovariance functions of the height distributions $h_{1}$ and $h_{2}$. The cross terms are zero since both distributions were supposed to be independent and of zero mean. Both autocovariance functions are again supposed to be isotropic and Gaussian:

$$
G(\tau)=\sigma_{1}^{2} \exp \left(-\tau^{2} /{T_{1}}^{2}\right)+{\sigma_{2}}^{2} \exp \left(-\tau^{2} / T_{2}^{2}\right) .
$$

And the power spectral density is

$$
\begin{aligned}
g(k)= & \pi{\sigma_{1}}^{2} T_{1}^{2} \exp \left(-k^{2} T_{1}^{2} / 4\right) \\
& +\pi{\sigma_{2}}^{2} T_{2}^{2} \exp \left(-k^{2} T_{2}^{2} / 4\right),
\end{aligned}
$$

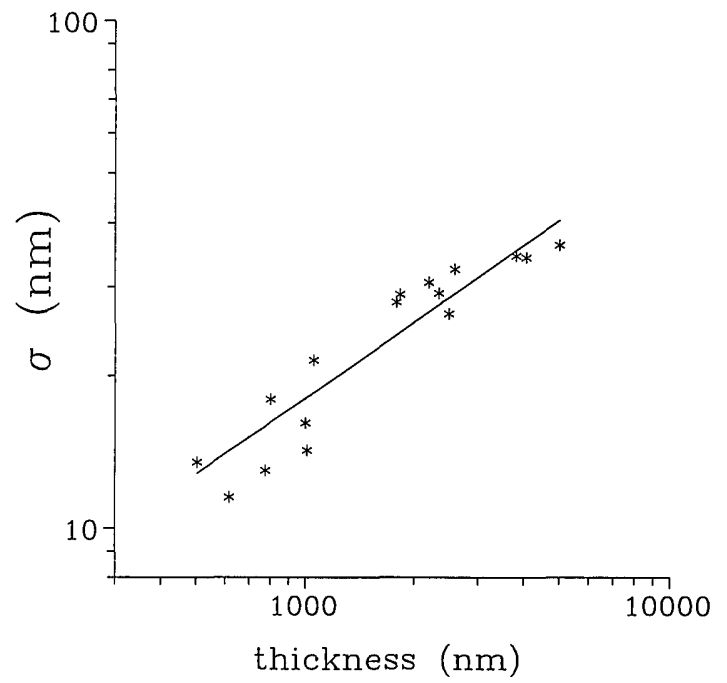

Fig. 9. Log-log plot of specific rms roughness of aluminum films versus thickness: *, calculated values from scattering measurements; solid line, fit of $\sigma$ to the square root of thickness.

which results in a separate contribution to scattering of both microstructures. Equation (16) means that the angular distribution of scattering is equal to the sum of the independent contributions of both microstructures.

According to Eq. (16) the specific roughness parameters of aluminum films, separated from those of the substrate, can be calculated from the angular distribution of scattering. Taking $\sigma_{1}=\sigma_{g}$ and $T_{1}=T_{g}$ as the substrate roughness parameters and calculating the specific autocorrelation length of aluminum by means of Eq. (9), one can calculate the specific rms roughness of aluminum by using a Monte Carlo method, similar to the one described in Subsection 4.B.1 with only one unknown: $\sigma$ of the aluminum film. This calculation is made for film thicknesses greater than $500 \mathrm{~nm}$, since for these mirrors TIS is sensibly larger

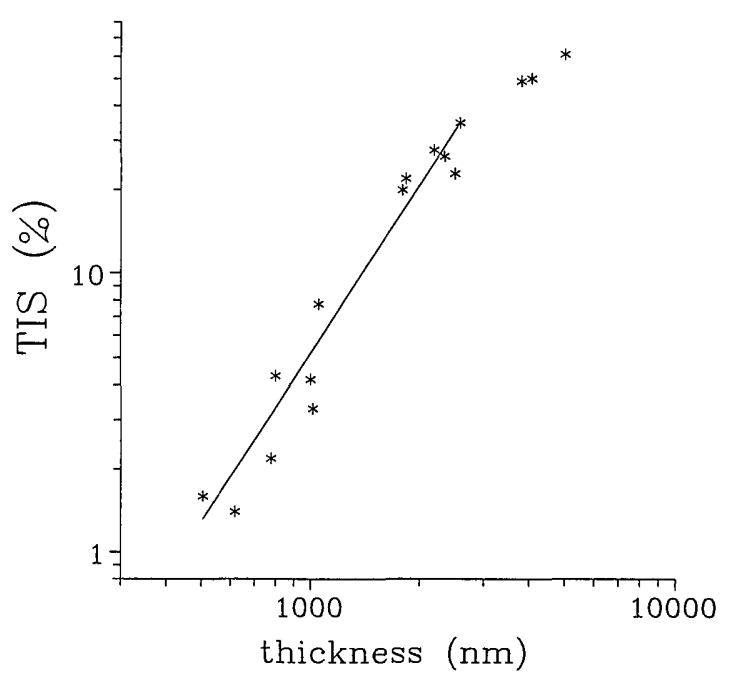

Fig. 10. Log-log plot of specific TIS of aluminum films versus aluminum thickness: *, calculated values from measurements of angular distribution of scattering; solid line, fit of TIS to the square of the thickness. 


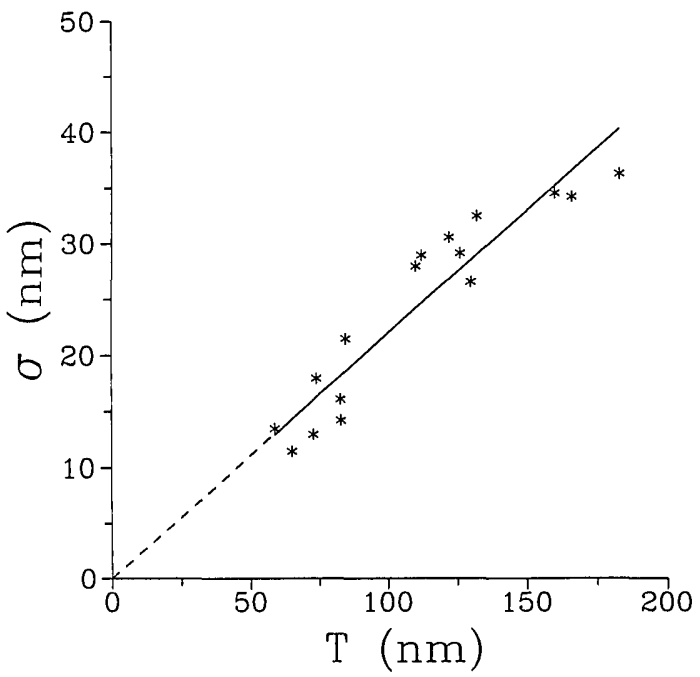

Fig. 11. Plot of rms roughness versus the autocorrelation length of aluminum films: *, calculated values from measurements of angular distribution of scattering and SEM pictures; solid line, fit of $\sigma$ proportional to $T$; dashed line, extrapolation proposed for thinner films.

than TIS $_{g}$. Figures 9 and 10 represent the calculated values of $\sigma$ and TIS aluminum specific parameters versus thickness, respectively. Figure 11 shows the relation between $\sigma$ and $T$.

Again, simple and reasonably good fits with oneparameter functions can be obtained to relate $\sigma$ and TIS with thickness and $\sigma$ with $T$ :

$$
\begin{aligned}
\sigma= & 0.57 \sqrt{e} \quad(e \text { and } \sigma \text { in nanometers }), \\
\text { TIS }= & 5.2 \times 10^{-6} e^{2} \\
& (e \text { in nanometers, TIS in percent }), \\
\sigma= & 0.22 T \quad(\sigma \text { and } T \text { in nanometers }) .
\end{aligned}
$$

Equation (19) shows that aluminum crystals grow proportionally in both dimensions (this proportionality was also obtained with other techniques such as microdensitometry ${ }^{7,9}$ and reflectance measurements ${ }^{10}$ for deposits of $\mathrm{Au}, \mathrm{Mg}, \mathrm{Ag}$, and $\mathrm{Cu}$ ). It can be thought that this proportion remains valid for thinner films (but thick enough to form a full layer).

\section{Conclusions}

The surface roughness parameters of thin aluminum films versus their thickness were obtained from measurements of the angular distribution of scattering and of the grain lateral size from SEM pictures. A first-order perturbation vector scattering theory was used in the calculations. The surface profile is supposed to have a Gaussian autocovariance function. The outer surface profile of aluminum film was determined by the microstructure of the substrate and that of aluminum crystals. The specific aluminum surface-roughness parameters were obtained, assuming that the height distribution function associated with the aluminum film is independent from that of the substrate. Simple empirical expressions relating surface-roughness parameters, TIS, and thickness were obtained.

\section{References}

1. G. Hass, W. R. Hunter, and R. Tousey, "Reflectance of evaporated aluminum in the vacuum ultraviolet," J. Opt. Soc. Am. 46, 1009-1012 (1956).

2. L. Holland, "Effect of vapor incidence on the structure of evaporated aluminum films," J. Opt. Soc. Am. 43, 376-380 (1953).

3. M. J. Verkerk and W. A. M. C. Brankaert, "Optical properties of reactively evaporated aluminum films," J. Mater. Sci. Lett. 6, 115-117 (1987).

4. J. G. Endriz and W. E. Spicer, "Study of aluminum films. I. Optical studies of reflectance drops and surface oscillations on controlled-roughness films," Phys. Rev. B 4, 4144-4159 (1971).

5. G. Brown, V. Celli, M. Haller, A. A. Maradudin, and A. Marvin, "Resonant light scattering from a randomly rough surface," Phys. Rev. B 31, 4993-5005 (1985).

6. J. M. Elson, "Light scattering from semi-infinite media for non-normal incidence," Phys. Rev. B 12, 2541-2542 (1975).

7. G. Rasigni, F. Varnier, M. Rasigni, J. P. Palmari, and A. Llebaria, "Autocovariance function, root-mean-square-roughness height and autocovariance length for rough deposits of copper, silver and gold," Phys. Rev. B 25, 2315-2323 (1982).

8. J. P. Rossi and D. Maystre, "Rigorous numerical study of speckle patterns for two-dimensional, random microrough surfaces," Opt. Eng. 25, 613-617 (1986).

9. M. Rasigni, G. Rasigni, J. P. Palmari, and A. Llebaria, "Study of surface roughness using a microdensitometer analysis of electron micrographs of surface replicas. II: Autocovariance functions," J. Opt. Soc. Am. 71, 1230-1237 (1981).

10. T. F. Gesell, E. T. Arakawa, M. W. Williams, and R. N. Hamm, "Optical and photoelectric properties of $\mathrm{Mg}$ in the vacuum ultraviolet," Phys. Rev. B 7, 5141-5146 (1972). 